Supporting Information for

\title{
A Planar Fully Stretchable Lithium Ion Batteries Based on Lamellar Conductive Elastomer
}

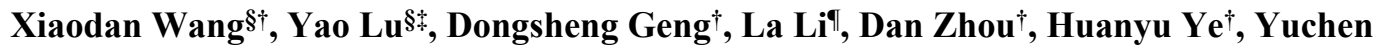 \\ Zhu ${ }^{\dagger}$, Rongming Wang*†
}

†Beijing Advanced Innovation Center for Materials Genome Engineering, Beijing

Key Laboratory for Magneto-Photoelectrical Composite and Interface Science,

School of Mathematics and Physics, University of Science and Technology Beijing, Beijing 100083, People's Republic of China

\#State Key Laboratory of Automotive Safety and Energy, Tsinghua University, Beijing 100084, China

IState Key Laboratory for Superlattices and Microstructures Institute of Semiconductors, Chinese Academy of Sciences, Beijing 100083, China.

*Corresponding Author : Prof. R.Wang. E-mail: rmwang@ustb.edu.cn 
1. The surface morphology of stretchable PVDF/TPU-Cu@Ag conductive substrate assisted with SEM.

The surface morphology of PVDF/TPU-Cu@Ag conductive substrate is analysed before electrode preparation. The results are shown in Figure S1.

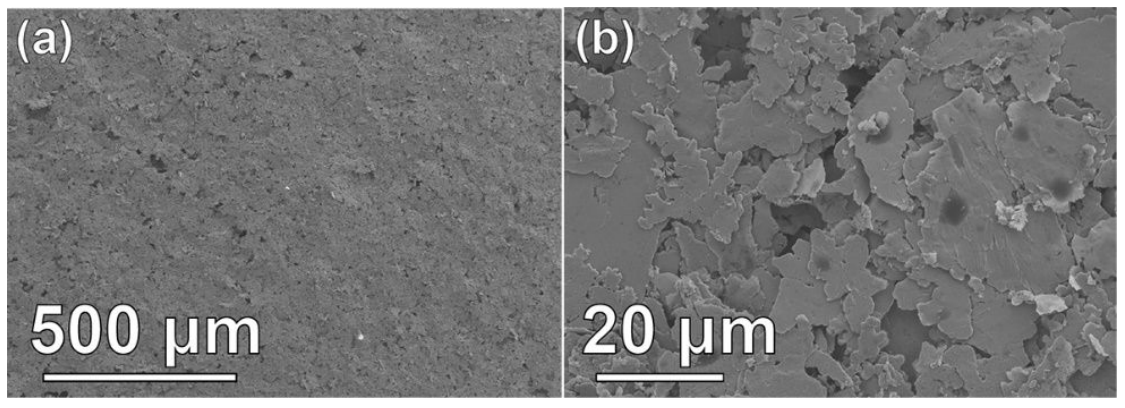

Figure S1 (a,b) SEM images of the TPU-Cu@Ag conductive substrate at different magnification. 
2. The conductivity of TPU-Cu@Ag conductive substrate.

The conductivity of TPU-Cu@Ag conductive substrate was measured at both released and stretched state. The dates were obtained by M-3 type four-probe tester which is shown in Figure S2

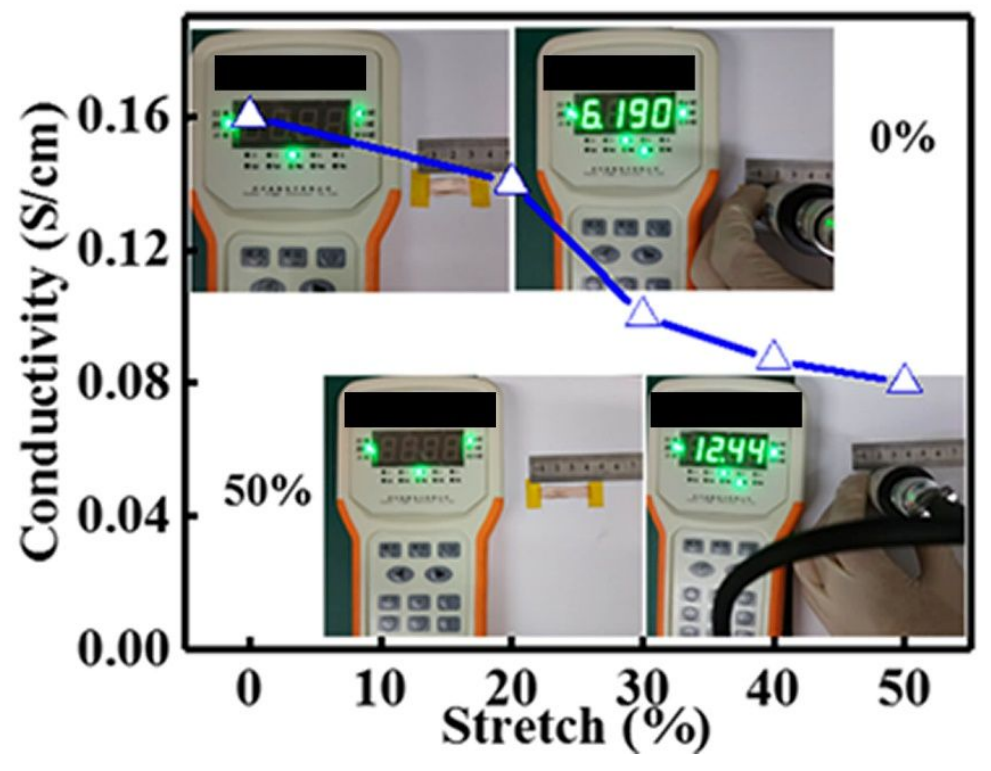

Figure S2 The relationship between conductivity and stretch ratio of TPU-Cu@Ag conductive substrate. 


\section{Comparison of conductivity of different conductive substrates.}

Three kinds of conductive substrates including TPU-Cu, TPU-Al and TPU-Cu@Ag were prepared to compare their conductivity. Only the TPU-Cu@Ag conductive substrate possesses excellent electrical conductivity due to the sheet structure of $\mathrm{Cu} @ \mathrm{Ag}$ powders.

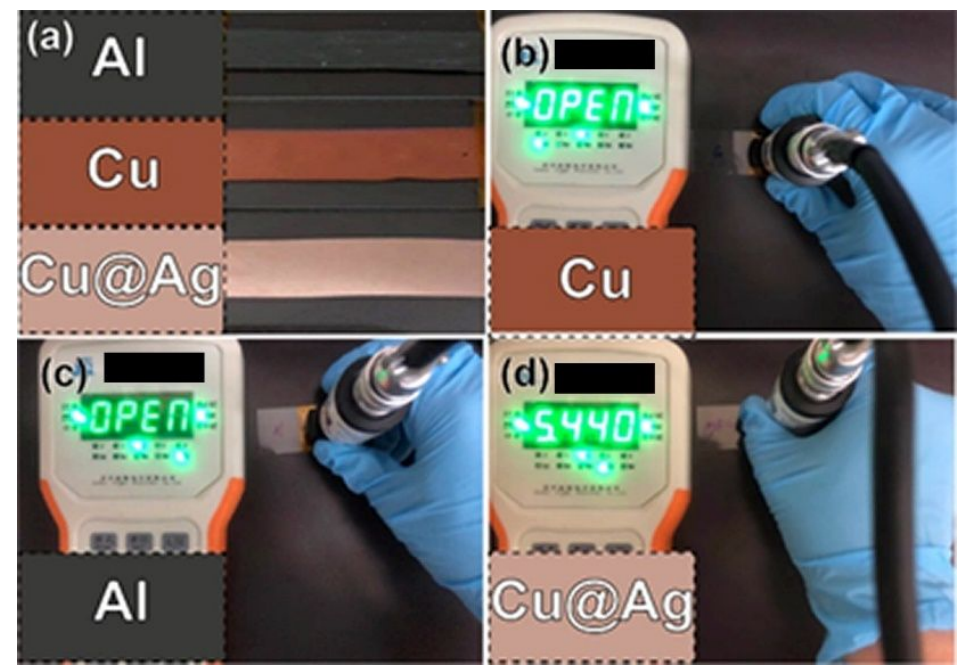

Figure S3 The conductivity comparison of TPU-Cu, TPU-Al and TPU-Cu@Ag substrate. (a) The digital photos of TPU-Cu, TPU-Al and TPU-Cu@Ag substrate. (b) The "OPEN" resistance value of TPU-Cu and (c) TPU-Al substrate displayed on resistance testing equipment. (d) The $5.4 \Omega$ resistance value of TPU-Cu@Ag substrate. 


\section{Stretchability of TPU/PVDF separator.}

The prepared TPU/PVDF separator can be stretched to $125 \%$ of initial length, the results are displayed in Figure S4.

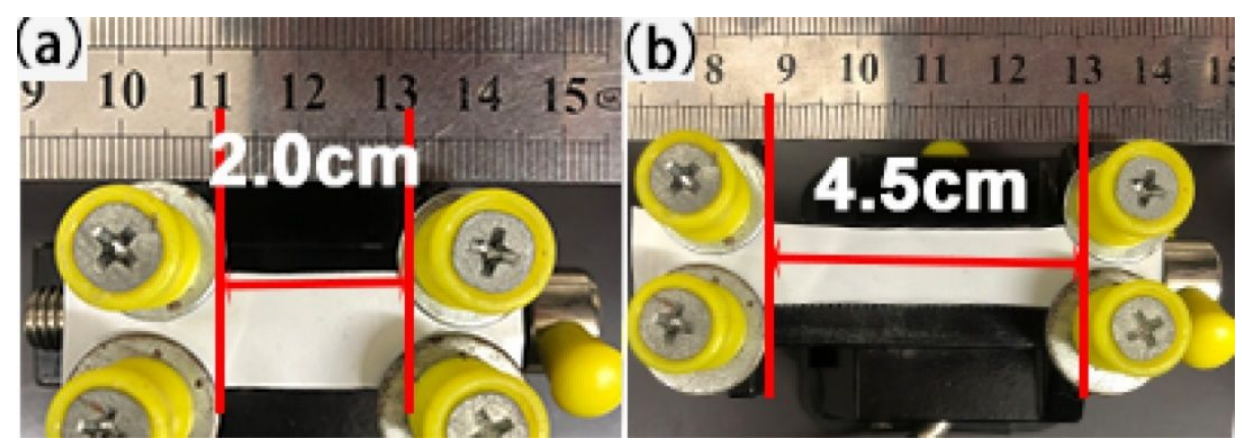

Figure S4 (a) The pristine state and (b) the $125 \%$ stretchable state of TPU/PVDF separator. 
5. Measuring the precursor surface topography of Si@C HFs.

From the Figure S5a, b, the independent organic fibers are observed clearly and the Si nanoparticles cluster can be found through the transparent organic fibers.
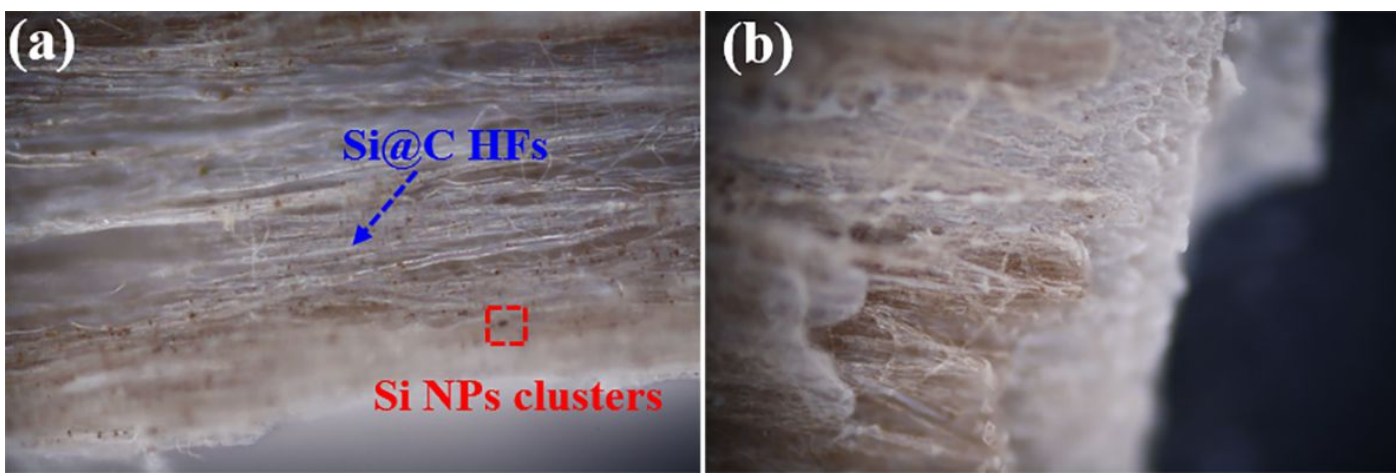

Figure S5 (a) The precursor optical microscope image and (b) the section image of core-shell Si@C HFs. 
Table S1

Fabricated Batteries By

Peng's group

Cui's group

Rogers' group

Our work
Comparison of Different Stretchable Batteries

Stretchability (\%)

Cycle numbers

Capacity $\left(\mathrm{mAh} / \mathrm{cm}^{2}\right)$

400

50

300

150

0.12

3.6

1.1

$20 \quad 1.1$

100

6 
